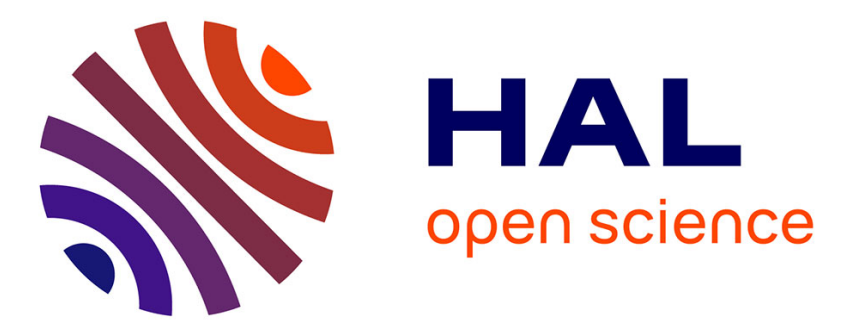

\title{
Topology preserving warping of 3-D binary images according to continuous one-to-one mappings
}

Sylvain Faisan, Nicolas Passat, Vincent Noblet, Renée Chabrier, Christophe Meyer

\section{- To cite this version:}

Sylvain Faisan, Nicolas Passat, Vincent Noblet, Renée Chabrier, Christophe Meyer. Topology preserving warping of 3 -D binary images according to continuous one-to-one mappings. IEEE Transactions on Image Processing, 2011, 20 (8), pp.2135-2145. 10.1109/TIP.2011.2158338 . hal-01694410

\section{HAL Id: hal-01694410 \\ https://hal.univ-reims.fr/hal-01694410}

Submitted on 26 Feb 2018

HAL is a multi-disciplinary open access archive for the deposit and dissemination of scientific research documents, whether they are published or not. The documents may come from teaching and research institutions in France or abroad, or from public or private research centers.
L'archive ouverte pluridisciplinaire HAL, est destinée au dépôt et à la diffusion de documents scientifiques de niveau recherche, publiés ou non, émanant des établissements d'enseignement et de recherche français ou étrangers, des laboratoires publics ou privés. 


\title{
Topology preserving warping of 3-D binary images according to continuous one-to-one mappings
}

\author{
S. Faisan*, N. Passat, V. Noblet, R. Chabrier, C. Meyer
}

\begin{abstract}
The estimation of one-to-one mappings is one of the most intensively studied topics in the research field of non-rigid registration. Although the computation of such mappings can now be performed accurately and efficiently, the solutions for using them in the context of binary image deformation is much less satisfactory. In particular, warping a binary image with such transformations may alter its discrete topological properties if common resampling strategies are considered. In order to deal with this issue, this article proposes a method for warping such images according to continuous and bijective mappings, while preserving their discrete topological properties (i.e. their homotopy type). Results obtained in the context of atlas-based segmentation of complex anatomical structures highlight the advantages of the proposed approach.
\end{abstract}

\section{Index Terms}

Binary image warping, discrete topology preservation, one-to-one mapping, simple points and skull segmentation.

S. Faisan, N. Passat, and V. Noblet are with the LSIIT UMR-CNRS UdS 7005. E-mail: faisan@ensps.u-strasbg.fr; vincent.noblet@unistra.fr; passat@dpt-info.u-strasbg.fr. Mailing address: ENSPS LSIIT - Bd Sébastien Brant - 67412 Illkirch, France. Phone: + 33368854489 - Fax: + 33368854497.

R. Chabrier is with the FRE 3289 CNRS UdS. E-mail: renee.chabrier@linc.u-strasbg.fr. Mailing address: IPB - 4, rue Kirschleger - 67085 Strasbourg, France. Phone: + 33368854037 - Fax: + 33368854087.

C. Meyer is with the Li4S - EA 4268 - IFR 133 - INSERM. E-mail: christophe.meyer@chu-besancon.fr. Mailing address: CHU de Besançon - Hôpital Jean Minjoz - Boulevard Fleming - 25030 Besançon - France. Phone: + 33381668234 - Fax: +33381668357 . 


\section{INTRODUCTION}

\section{A. Motivation}

During the past two decades, many methods have been proposed to non rigidly register 3D images, enabling the estimation of deformation fields, that model the geometrical variations between two images presenting a similar semantics. An intensive effort has been devoted to develop registration methods yielding deformation fields that are one-to-one mappings, thus preserving the topology of the warped image in the continuous domain (see [1] for a survey). Particularly in the context of inter-subject medical image registration, such constraint appears to be relevant since, in the continuous domain, anatomical structures have the same topology for any individual (at least for non-pathological cases). Topology preservation ensures that connected structures remain connected and that the neighborhood relationship between structures is maintained. It also prevents the appearance or disappearance of new or existing structures. Notice that the existing topology preserving registration methods are generally developed in the "continuous" space of the support of the image (i.e. a part of $\mathbb{R}^{3}$ ).

However, applying such deformation fields to warp a discrete image (i.e. defined on a part of $\mathbb{Z}^{3}$ ) may raise some problems, especially for images defined with a limited number of values, such as binary or label images. For instance, in the context of atlas-based segmentation, warping a predefined atlas of anatomical structures according to a deformation field that ensures topology preservation in the continuous domain may not guarantee that the discrete topology of the warped atlas is preserved. Consequently, all the "good" properties induced by such deformation fields (i.e. no splitting, no merging, etc.) may definitely be lost when actually applied on binary or label images.

Applying such deformation fields in a "correct" way, i.e. by guaranteeing that the topology of the warped discrete objects is not altered, is of precious use for several purposes including (but not limited to):

- visualisation of anatomical structures: topology errors may generate visualisation artifacts, e.g. when generating a mesh model of a surface;

- positioning of an initial model as the preliminary step of a topology preserving deformable model method;

- analysis of segmented structures: topological details, such has holes or cavities can actually be used as landmarks;

- post-processing of segmented structures: for instance, the -classical- computation of differential properties of a cortical surface will be incorrect if this surface is not actually a manifold. 
In such a crucial context, it is not possible to consider standard interpolation methods (which provide no topological guarantee) to perform discrete object warping based on topology preserving deformation fields. More sophisticated processes, such as the one proposed in this article, are then necessary.

\section{B. Problem statement}

Image warping is the process of applying a geometric transformation to a discrete image. Two different ways can be considered to define a transformation for warping an image $M$ to obtain an image $T$. We can consider either the transformation that associates for each point of $M$ its coordinates in $T$ or the transformation that associates for each point of $T$ its coordinates in $M$, the two transformations being the inverse one of each other in case of one-to-one mapping. The second formulation is used in this paper. This formulation is generally preferred since the first one would require the use of irregular sample interpolation methods, whereas the second one can rely on standard interpolation techniques. Given a discrete image $M$ and a continuous transformation $h$, the purpose is to compute the warped image $T$ so that for each voxel $v$ we have $T(v)=M \circ h(v)=M(h(v))$. The main problem is that, in the general case, $h(v)$ does not necessarily correspond with "grid points" of $M$. In such conditions, $M \circ h$ is not correctly defined. Some interpolation techniques are then required to consider a continuous representation of image $M$ which has the same values as the sampled data at the grid locations (exact interpolation).

Although several image interpolation techniques (linear, cubic) [2] have been proposed for graylevel images, no specific attention has been paid to the case of binary images. In particular, common interpolation techniques, except the nearest neighbor interpolation, do not guarantee the resampled image $T=M \circ h$ to remain a binary one. To circumvent this limitation, it is possible to perform a postprocessing thresholding operation to get a binary image. Unfortunately, warping a discrete image $M$ according to a continuous and bijective (i.e. topology-preserving) transformation $h$ with such common interpolation techniques may fail in preserving its discrete topological properties, i.e., $M$ and $T$ may not have the same homotopy type. As an illustration, a synthetic 2-D binary image, composed of one circle (see $M$ in Fig. 1), has been warped according to a one-to-one mapping (an affine transformation with a positive determinant) using the nearest neighbor interpolation. The topology of the resulting warped image $T^{(N)}$ is strongly altered: the hole of $M$ has been fused with the infinite background component of the image, while the circle has been split into several disconnected components (see right side of Fig. 1).

Two main strategies can be considered to warp a binary image $M$ according to a one-to-one mapping $h$, leading to a solution $T=M \circ h$ topologically equivalent to $M$. The first strategy is to compute the 


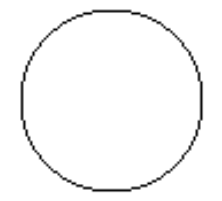

$M$

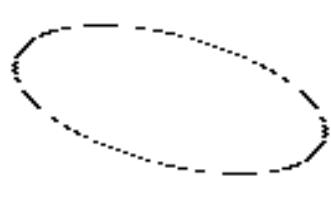

$T^{(N)}$

Fig. 1. Topological alterations induced by the nearest neighbor interpolation. The binary image $M$ is composed of one circle. The result $T^{(N)}$ of the warping of $M$ according to an affine transformation using the nearest neighbor interpolation is not topologically equivalent to $M$ (see text).

binary image $T$ by using a standard interpolation method with a post-processing thresholding operation, and to automatically correct the topology of $T$ a posteriori. However, nearly all the methods devoted to topology correction can only be applied to simply connected objects, in particular, full or hollow spheres [3], [4], [5], [6], [7], [8], [9], with applications to the segmentation of anatomical structures assumed to present such non-complex topologies (e.g. cortex or vascular trees). The correction of objects presenting more complex topologies is still an open problem in 3-D. To the best of our knowledge, the most general methods proposed in the literature are for hole closing [10] (which enables to choose the size of the holes to be closed), and a dual approach for holes retrieval [11] (which enables to choose the number and size of the holes to retrieve).

An alternative strategy consists in preserving the topological properties of the object during the whole warping process, thus guaranteeing the initial and final objects to be topologically equivalent. The basic idea is to start from $T=M$, and to modify $T$ in a homotopy-preserving fashion until $T$ is geometrically as close as possible to $M \circ h$.

This paradigm based on the modification of a binary image by preserving its discrete topology is generally considered in the context of segmentation. Such segmentation methods [12], [13], [14], [15], [16] often rely on the concept of 3-D simple points [17], i.e. points whose addition or removal to/from a binary object does not alter its topology and in particular preserves its homotopy type. Such points are added and/or removed to/from an initial model, having the desired topology w.r.t. the structure to segment, the choice of the points being generally guided by constraints related to anatomical and/or physical properties of the structure. Note that, since simple points can be locally characterized in constant time [18], such methods are often of low algorithmic complexity. We can notice finally that few methods also propose to use other topological modeling frameworks such as cubical complexes (see e.g. [19]).

To the best of our knowledge, the only method tackling the problem of warping a binary image by 
preserving its discrete topology has been proposed in [20]. More precisely, this approach is devoted to label images. However, we will only consider it in the context of binary image warping. A justification of this restriction is provided in Section V. The basic idea of this method is to modify the initial image $M$ in a homotopy-preserving fashion by "moving" simple points in the direction provided by the continuous deformation field $h$. This continuous one-to-one mapping is approximated by a digital homeomorphism $h_{d}$, which is directly estimated from the movement of simple points. The algorithm ends when the residual error for each voxel $v$ verifies $\left\|h(v)-h_{d}(v)\right\|<1$ (i.e. the "size" of a voxel) or when no more voxel can be moved. Although this method ensures topology preservation, it may fail to converge to the geometrically desired solution. Indeed, in the context of brain tissue segmentation warping, [20] reports maximal error higher than 20 voxels between the estimated digital homeomorphism and the continuous transformation. We also highlight such problems of convergence in experiments presented in Section III.

This article presents a new strategy to tackle this problem, whose preliminary results have been published in [21]. We propose here to formulate the problem of warping a binary image according to a continuous deformation field in an optimization framework. Such framework requires to define a cost function, that should be minimal for the desired solution, and an optimization strategy, that should converge to the global minimum. Similarly to [20], we use a dynamical scheme based on the addition and/or removal of simple points. This scheme is guided by the proposed cost function, which is minimal when the estimated discrete image $T$ is as close as possible to $M \circ h$. An efficient optimization strategy has also been proposed. This framework is more comprehensive than the one proposed in [20], which lacks of formalization, thus being difficult to extend or to improve. Moreover, directly estimating the warped image $T$ rather than the corresponding digital homeomorphism $h_{d}$ as in [20] enables to reduce the dimensionality of the search space (estimating $h_{d}$ requires 3 times more parameters), and thus may be a more efficient strategy, that may be less likely caught in local minima. Notice that, when the warped image $T$ is successfully estimated, a digital homeomorphism $h_{d}$, that approximates at best $h$, can straightforwardly be obtained by associating to each voxel $v$ of $T$ the voxel $v^{\prime}$ of $M$ that belongs to the same connected component of $v$ and that is as close as possible to $h(v)$.

The article is organized as follows. In Section II, the proposed method is described. Section III summarizes experimental results in the context of atlas-based segmentation of a complex anatomical structure, namely the skull. Discussions and conclusions are provided in Sections IV and V, respectively. 


\section{METHOD}

Let $E_{\mathbb{R}}^{M}, E_{\mathbb{R}}^{T} \subset \mathbb{R}^{3}$. Let $E_{\mathbb{Z}}^{M}, E_{\mathbb{Z}}^{T} \subset \mathbb{Z}^{3}$ such that $E_{\mathbb{Z}}^{M}=E_{\mathbb{R}}^{M} \cap \mathbb{Z}^{3}$ and $E_{\mathbb{Z}}^{T}=E_{\mathbb{R}}^{T} \cap \mathbb{Z}^{3}$. The sets $E_{\mathbb{Z}}^{M}$ and $E_{\mathbb{Z}}^{T}$ are devoted to provide a support for digital images, while the sets $E_{\mathbb{R}}^{M}$ and $E_{\mathbb{R}}^{T}$ are parts of the Euclidean space devoted to provide a support for their associated continuous analogues (see Subsection II-A).

The process of warping a binary image $M: E_{\mathbb{Z}}^{M} \rightarrow\{0,1\}$ according to a continuous and bijective transformation $h: E_{\mathbb{R}}^{T} \rightarrow E_{\mathbb{R}}^{M}$ to obtain a warped image $T: E_{\mathbb{Z}}^{T} \rightarrow\{0,1\}$ amounts to find, among the set of binary images topologically equivalent $(\sim)$ to $-i$.e. having the same homotopy type as- $M$, the optimal image $\hat{T}$, that minimizes the following constrained optimization problem:

$$
\hat{T}=\arg \min _{T \sim M} d(T, M, h)
$$

where $d(T, M, h)$ is a distance between $T$ and $M \circ h$. However, as it has been mentioned in Section I, $M \circ h$ is not correctly defined and some interpolation techniques are then required to evaluate a continuous image $M_{\mathbb{R}}: E_{\mathbb{R}}^{M} \rightarrow\{0,1\}$ verifying the constraint: $\left(M_{\mathbb{R}}\right)_{\mid E_{\mathbb{Z}}^{M}}=M$. Such an image $M_{\mathbb{R}}$ enables to define $M_{\mathbb{R}} \circ h$, and then to compute $M \circ h$ as being $\left(M_{\mathbb{R}} \circ h\right)_{\mid E_{\mathbb{Z}}^{T}}$. Consequently, $d(T, M, h)$ is in fact a distance between $T$ and $M_{\mathbb{R}} \circ h$.

The basic idea to solve this constrained optimization problem is the following: $T$ is first initialized with $M$ and is then modified by adding and removing simple points so that to minimize the distance between $T$ and the continuous warped image $M_{\mathbb{R}} \circ h$. To avoid convergence to local minima, the problem is solved in an incremental way by considering a decomposition of the transformation $h$ into small displacements fields. A global overview of the method is provided in Algorithm 1.

After preliminary considerations in Subsection II-A related to the relation between the continuous and the discrete representations of an image, we introduce, in Subsection II-B, a convenient distance $d$. In Subsection II-C, we explain how to constrain $T$ to remain topologically equivalent to $M$ during the deformation process. Finally, the optimization strategy is detailed in Subsection II-D.

\section{A. From discrete to continuous representation}

In the proposed framework, we associate to any discrete binary image $X: E_{\mathbb{Z}}^{X} \rightarrow\{0,1\}$ (with $E_{\mathbb{Z}}^{X} \subset \mathbb{Z}^{3}$ ), the continuous binary image $X_{\mathbb{R}}: E_{\mathbb{R}}^{X} \rightarrow\{0,1\}$ obtained by associating to each point $x=\left(x_{1}, x_{2}, x_{3}\right) \in E_{\mathbb{Z}}^{X}$ the unit cubical cell $\left[x_{1}-1 / 2, x_{1}+1 / 2\right] \times\left[x_{2}-1 / 2, x_{2}+1 / 2\right] \times\left[x_{3}-1 / 2, x_{3}+\right.$ $1 / 2] \subset E_{\mathbb{R}}^{X}$. These cubical cells being closed sets, the boundaries of the continuous objects of $X_{\mathbb{R}}$ associated to those of $X$ also belong to these objects (which are then closed sets too). According to this 
(classical) discrete/continuous correspondence, the continuous binary image $X_{\mathbb{R}}$ has the same topology as the discrete binary image $X$, considered in a $(26,6)$-adjacency framework (i.e. the 26-adjacency for the "object part" of $X$, and the 6-adjacency for the "background part") [22]. The (26,6)-adjacency is used throughout the paper. Notice however that the methodological framework is -by definition- not sensitive to the adjacency duality. Indeed, warping a binary image using the $(6,26)$-adjacency can be obtained by warping the complement of the binary image (zero becomes one and one becomes zero) using the $(26,6)$-adjacency. We denote by $C[X]$ (resp. $C\left[X_{\mathbb{R}}\right]$ ) the set of all the connected components (CCs) of $X$ (resp. $X_{\mathbb{R}}$ ), i.e. both object CCs (OCCs) and background CCs (BCCs). There exists a straightforward bijection between the CCs of $C\left[X_{\mathbb{R}}\right]$ and those of $C[X]$, which associates to each connected component $K \in C\left[X_{\mathbb{R}}\right]$ its discrete analogue (namely $K \cap E_{\mathbb{Z}}^{X}$ ) in $C[X]$. In such conditions, it is possible to consider the computation of the distance $d$ only on the continuous representation of the considered images.

\section{B. Cost function}

Since $T_{\mathbb{R}}$ and $M_{\mathbb{R}}$ are assumed to be topologically equivalent, there exists a bijection $\phi: C\left[T_{\mathbb{R}}\right] \rightarrow$ $C\left[M_{\mathbb{R}}\right]$ between the CCs of $T_{\mathbb{R}}$ and $M_{\mathbb{R}}$. We define $c_{X_{\mathbb{R}}}: E_{\mathbb{R}}^{X} \rightarrow C\left[X_{\mathbb{R}}\right]$ as the function that associates to each point $v$ the $\mathrm{CC}$ in which it lies.

Let $v \in E_{\mathbb{R}}^{T}$. The point-to-set distance $\rho_{T_{\mathbb{R}}}\left(h(v), M_{\mathbb{R}}\right)$ can be defined as:

$$
\rho_{T_{\mathbb{R}}}\left(h(v), M_{\mathbb{R}}\right)=\min _{\left\{x \mid c_{M_{\mathbb{R}}}(x)=\phi\left(c_{T_{\mathbb{R}}}(v)\right)\right\}}\|x-h(v)\| .
$$

This represents the distance between $h(v)$ and the $\mathrm{CC} \phi\left(c_{T_{\mathbb{R}}}(v)\right)$ of $M_{\mathbb{R}}$ which is associated to the CC of $T_{\mathbb{R}}$ containing $v$. Note that, if $\phi\left(c_{T_{\mathbb{R}}}(v)\right)$ is equal to $c_{M_{\mathbb{R}}}(h(v))$, i.e. if $v$ and $h(v)$ belong to equivalent CCs of $M_{\mathbb{R}}$ and $T_{\mathbb{R}}$, then $\rho_{T_{\mathbb{R}}}\left(h(v), M_{\mathbb{R}}\right)=0$. The computation of $\rho_{T_{\mathbb{R}}}$ is illustrated in a 2-D case in Fig. 2.

From this point-to-set distance definition, we can derive a set-to-set distance between $M_{\mathbb{R}}$ and the image of $T_{\mathbb{R}}$ by $h$ as:

$$
\begin{aligned}
d\left(T_{\mathbb{R}}, M_{\mathbb{R}}, h\right) & =\int_{v \in E_{\mathbb{R}}^{T}} \rho_{T_{\mathbb{R}}}\left(h(v), M_{\mathbb{R}}\right) \cdot d v \\
& \approx \sum_{v \in E_{\mathbb{Z}}^{T}} \rho_{T_{\mathbb{R}}}\left(h(v), M_{\mathbb{R}}\right)
\end{aligned}
$$

We can notice that the distance $d\left(T_{\mathbb{R}}, M_{\mathbb{R}}, h\right)$ may not be defined for a result obtained with the nearest neighbor interpolation since its computation requires a one-to-one mapping $\phi$ between the CCs of $M$ and those of $T$. However, by setting (even if it is not the case) that object points and background points belong to the same OCC, and to the same BCC, respectively, the result obtained with the nearest neighbor interpolation enables to vanish the cost function under approximation of Eq. 3(ii). 


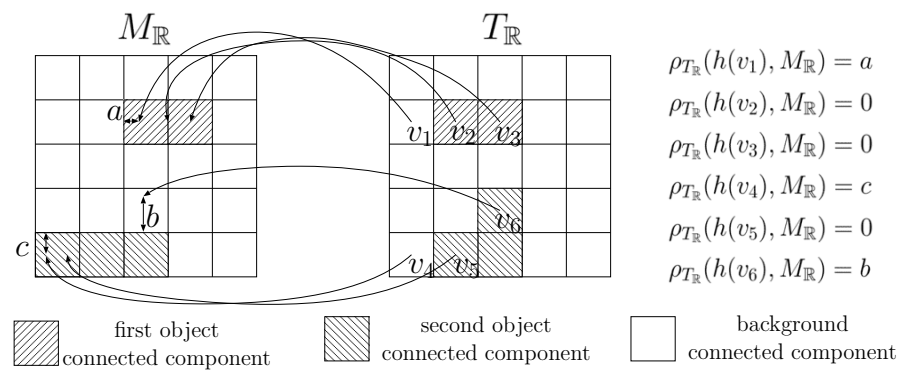

Fig. 2. Computation of $\rho_{T_{\mathbb{R}}}\left(h(),. M_{\mathbb{R}}\right)$ for 6 points. The image $M_{\mathbb{R}}$ is composed of two OCCs and one BCC. For instance, $\rho_{T_{\mathbb{R}}}\left(h\left(v_{2}\right), M_{\mathbb{R}}\right)=0$ since $v_{2}$ and $h\left(v_{2}\right)$ belong to equivalent CCs. However, since $v_{6}$ belongs to the second OCC of $T_{\mathbb{R}}$ whereas $h\left(v_{6}\right)$ belongs to the BCC of $M_{\mathbb{R}}$, we have $\rho_{T_{\mathbb{R}}}\left(h\left(v_{6}\right), M_{\mathbb{R}}\right)=b$, namely, the distance between $h\left(v_{6}\right)$ and the second OCC of $M_{\mathbb{R}}$.
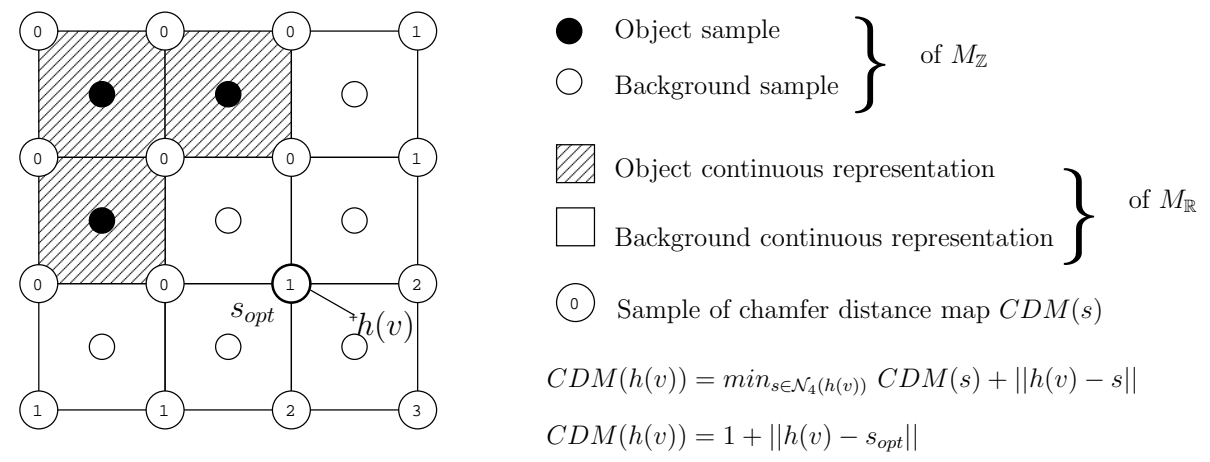

Fig. 3. Evaluation of the chamfer distance map $C D M$ at a non-discrete position $h(v)$ (in $2 \mathrm{D}$ case for the sake of clarity). Notice that the chamfer distance map is computed on a grid translated by a vector $[1 / 2,1 / 2]$ with respect to the sampling grid of image $M$, so that $C D M(s)$ corresponds to the distance toward the surface of the continuous representation $M_{\mathbb{R}}$.

The distance $d\left(T_{\mathbb{R}}, M_{\mathbb{R}}, h\right)$ does not exactly correspond to the distance between $M_{\mathbb{R}} \circ h$ and $T_{\mathbb{R}}$ but actually to the distance between $M_{\mathbb{R}}$ and $T_{\mathbb{R}} \circ h^{-1}$. This choice is motivated by the fact that the computation of $\rho_{T_{\mathbb{R}}}$ is done using distance maps in $M_{\mathbb{R}}$. As $M_{\mathbb{R}}$ does not evolve, the distance maps only need to be computed once for each CC. It would not be the case by considering a distance between $T_{\mathbb{R}}$ and $M_{\mathbb{R}} \circ h$ since the distance maps should have been computed in $T_{\mathbb{R}}$ and thus should have been updated whenever $T_{\mathbb{R}}$ is modified during the transformation scheme.

The term $\rho_{T_{\mathbb{R}}}\left(h(v), M_{\mathbb{R}}\right)$ is approximated by considering the chamfer distance map in $M$ to the $\mathrm{CC} \phi\left(c_{T_{\mathbb{R}}}(v)\right)$ since it provides a very satisfying approximation of the Euclidian distance map with a very low algorithmic cost. Evaluation of the chamfer distance map $(C D M)$ at a non-discrete position 
$h(v)$ is then done as follows (see Fig. 3): if $h(v)$ does not belong to $\phi\left(c_{T_{\mathbb{R}}}(v)\right), C D M(h(v))$ is set to $\min _{s \in \mathcal{N}_{8}(h(v))} C D M(s)+\|h(v)-s\|, \mathcal{N}_{8}(h(v))$ being the 8 nearest neighbor voxels of $h(v)$, else $C D M(h(v))$ is set to 0 . Notice that the chamfer distance map should be computed carefully in order to represent distance to the boundaries of the continuous $\mathrm{CC}$ and not to the discrete $\mathrm{CC} \phi\left(c_{T_{\mathbb{R}}}(v)\right) \cap E_{\mathbb{Z}}^{M}$. One solution is to compute the chamfer map on a grid whose points are located on the surface of the CCs of $M_{\mathbb{R}}$. Such a grid can be obtained by translating the former one from the vector $[1 / 2,1 / 2,1 / 2]$ (see Fig. 3). Notice that the chamfer distance maps need only to be computed once for each CC.

\section{Topology handling}

The cost function has been defined from the continuous representation of the images. However, we consider hereafter only their discrete representations. As in the continuous case, we denote by $c_{X}: E_{\mathbb{Z}}^{X} \rightarrow$ $C[X]$ the function that associates to each point $v$ the $\mathrm{CC}$ in which it lies.

To constrain $T$ to remain topologically equivalent to $M$ during the deformation process, $T$ is initialized to $M$ and is then modified by iterative removal/addition of simple points. Changing the value of a simple point $v$ in $T$ requires to determine its new corresponding $\mathrm{CC}$ to compute the cost function. This can be done unambiguously, since $v$ is a simple point, and is then adjacent to exactly one OCC (in the 26neighborhood) and one BCC (in the 6-neighborhood). For instance, in Fig. 2, if the value of the simple point $v_{1}$ of $T$ is modified, the CC associated to $v_{1}$ will be the first OCC $\left(c_{T}\left(v_{1}\right)\right.$ will then be modified in consequence). The function $c_{T}$ is stored and updated throughout the algorithm.

The value of a simple point in $T$ is modified only if it decreases the cost function. To this end, it is sufficient to compute, for each simple point $v$, the "benefit" to modify its value. This benefit is defined by:

$$
\begin{aligned}
b\left(v, T, M_{\mathbb{R}}, h\right) & =d\left(T_{\mathbb{R}}, M_{\mathbb{R}}, h\right)-d\left(T_{\mathbb{R}}^{\prime}, M_{\mathbb{R}}, h\right) \\
& =\rho_{T_{\mathbb{R}}}\left(h(v), M_{\mathbb{R}}\right)-\rho_{T_{\mathbb{R}}^{\prime}}\left(h(v), M_{\mathbb{R}}\right),
\end{aligned}
$$

where $T^{\prime}$ is the image obtained from $T$ by modifying the value at $v$.

An important property of the benefit function is that, when modifying a simple point $v$ in $T$ to obtain a new image $T^{\prime}$, we have:

$$
b\left(x, T, M_{\mathbb{R}}, h\right)=b\left(x, T^{\prime}, M_{\mathbb{R}}, h\right)
$$

for all simple points $x \neq v$ of $T$ and $T^{\prime}$, and

$$
b\left(v, T, M_{\mathbb{R}}, h\right)=-b\left(v, T^{\prime}, M_{\mathbb{R}}, h\right) .
$$


Consequently, when changing the value of $T$ at $v$, there is no need to update the benefit function $b$ for points which are simple in both images $T^{\prime}$ and $T$. Notice that the benefit function $b\left(v, T, M_{\mathbb{R}}, h\right)$ can only be evaluated for simple points $v$ of $T$ since its computation requires to change the value at voxel $v$. In particular, if $v$ were not a simple point, $T^{\prime}$ and $T$ would possibly not have the same topology, then it may be impossible to compute $\rho_{T_{\mathbb{R}}^{\prime}}\left(h(v), M_{\mathbb{R}}\right)$ since $\phi\left(c_{T^{\prime}}(v)\right)$ may not be defined unambiguously.

\section{Optimization strategy}

The purpose of the optimization strategy is to find an image $T$ that minimizes the cost function (Eq. 3) while being topologically equivalent to $M$. Starting from $T=M, T$ is then modified by iterative removal/addition of simple points. $T$ is also constrained to remain topologically equivalent to $M$ during the optimization algorithm. Moreover, in order to minimize the cost function, i.e. to converge to a geometrically desired solution, the optimization strategy consists in iteratively modifying the value of $T$ at the simple point of highest benefit $b$ until convergence. The implementation is based on an ordered list $\mathcal{L}$ which contains all simple points of $T$ characterized by a positive benefit value. If $v_{0}$ denotes the "first" point of $\mathcal{L}$, i.e. the simple point of $T$ of highest benefit, the value of $T$ at $v_{0}$ is modified $\left(T\left(v_{0}\right)=1-T\left(v_{0}\right)\right)$. The ordered list has then to be updated accordingly. Due to Eq. $6, v_{0}$ must be removed from $\mathcal{L}$. Moreover, since there is no need to update the benefit function for points which are simple in both images, i.e., for the images obtained before and after the modification at $v_{0}$ (Eq. 5), only points which became simple or non-simple after the modification at $v_{0}$ have to be considered to update $\mathcal{L}$. Modifying the value of $T$ at $v_{0}$ may only change the status of the simple points which are in the 26-neighborhood of $v_{0}$ : points which were not simple and which become simple must be added in $\mathcal{L}$ if they have a positive benefit value whereas points which were in $\mathcal{L}$ and which become non-simple must be removed from $\mathcal{L}$. The algorithm ends when $\mathcal{L}$ is empty, i.e. when all points are non-simple or have a non-positive benefit value. Unfortunately, this strategy suffers from some limitations which led us to propose two major improvements.

A first limitation of the method is illustrated in Fig. 4. To converge to the desired result $T^{\prime \prime}$ (global minimum) from the current image $T$, the label of $v_{2}$ has first to be changed to obtain $T^{\prime} . T^{\prime \prime}$ can then be obtained by modifying the label at $v_{1}$. However, the process may fail to converge to $T^{\prime \prime}$ if the first modification increases the cost function $\left(b\left(v_{2}, T, M_{\mathbb{R}}, h\right)<0\right)$, leading to refuse this operation, whereas both modifications may decrease the cost function: $b\left(v_{1}, T^{\prime}, M_{\mathbb{R}}, h\right)+b\left(v_{2}, T, M_{\mathbb{R}}, h\right)>0$.

In order to avoid such deadlock situations, we authorize the pairwise -topology-preserving- value modification of two neighbor points, $v_{1}$ and $v_{2}$, provided that it decreases the cost function. This process 


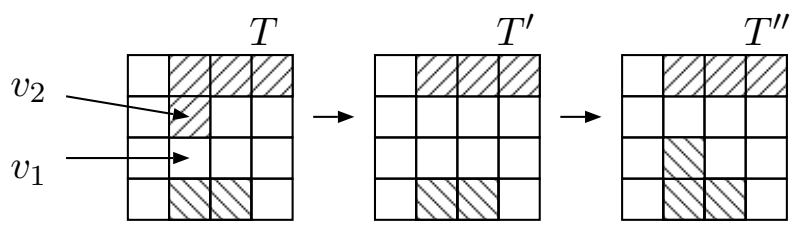

(a)

(b)

(c)

Fig. 4. Illustration of a deadlock situation justifying the usefulness of pairwise topology-preserving modification (see text).

is achieved only when $\mathcal{L}$ is empty, namely, when there is no more simple point with positive benefit. Under this hypothesis, it can be proven that such voxels $v_{1}$ and $v_{2}$ can be found by considering only non-simple points $v_{1}$ verifying $\rho_{T_{\mathbb{R}}}\left(h\left(v_{1}\right), M_{\mathbb{R}}\right)>0$. For such a point $v_{1}$, every simple points $v_{2}$ in its neighborhood are considered as potential candidates for pairwise modification. Each of these voxels is temporarily changed to obtain $T^{\prime}$. Only voxels $v_{2}$ that enable $v_{1}$ to become a simple point in $T^{\prime}$ are retained. The modification of the values at voxels $v_{1}$ and $v_{2}$ is then performed if it actually reduces the cost function, i.e. if $b\left(v_{1}, T^{\prime}, M_{\mathbb{R}}, h\right)+b\left(v_{2}, T, M_{\mathbb{R}}, h\right)>0$. If several points $v_{2}$ verify these conditions, the voxel which permits to decrease at best the cost function is chosen. This process may generate new simple points in the neighborhood of the involved points, enabling to keep deforming the current image by "classical" simple point modification.

A second improvement aims at avoiding convergence to local minima, which might happen especially with "large" displacements. The basic idea is to perform transformation in an incremental way by considering $N+1$ intermediate transformations, $h^{(0)}, h^{(1)}, \ldots, h^{(N)}$, computed from $h$ such that:

$$
\left\{\begin{array}{l}
h^{(0)}=I d \\
\forall j \in[0, N-1], \forall v \in E_{\mathbb{Z}}^{T},\left\|h^{(j+1)}(v)-h^{(j)}(v)\right\|<\epsilon \\
h^{(N)}=h,
\end{array}\right.
$$

thus leading to small displacements. The optimization scheme described above is achieved by considering sequentially $h^{(1)}, h^{(2)}, \ldots, h^{(N)}$. These transformations enable to guide the discrete dynamical process to the desired solution. The smaller $\epsilon$ is, the more the dynamical process is guided, and the better the convergence properties of the algorithm are. However, it is not worth using too small values for $\epsilon$ since the dynamical process is discrete. In the experiments, $\epsilon$ has been set to 0.5 which has been shown to be a relevant choice at the sight of the results reported in Subsection III-C investigating the influence of $\epsilon$. An easy way to estimate intermediate transformations $h^{(i)}(0<i<N)$ verifying Eq. 7 is to use the 
following linear decomposition:

$$
\forall v \in E_{\mathbb{Z}}^{T}, h^{(i)}(v)=v+\frac{i}{N}(h(v)-v),
$$

where $N$ is the smallest integer such that: $N \geq \max _{v \in E_{\mathbb{Z}}^{T}}\|h(v)-v\| / \epsilon$.

A global overview of the method is given in Algorithm 1. Notice finally that, since the distance between $T$ and $M \circ h$ is enforced to decrease across the iterations, and since there are a finite number of voxels (and consequently a finite number of binary images), the procedure is ensured to converge. Unfortunately, there is no guarantee of convergence to the global minimum since the optimization scheme might be trapped in local minima.

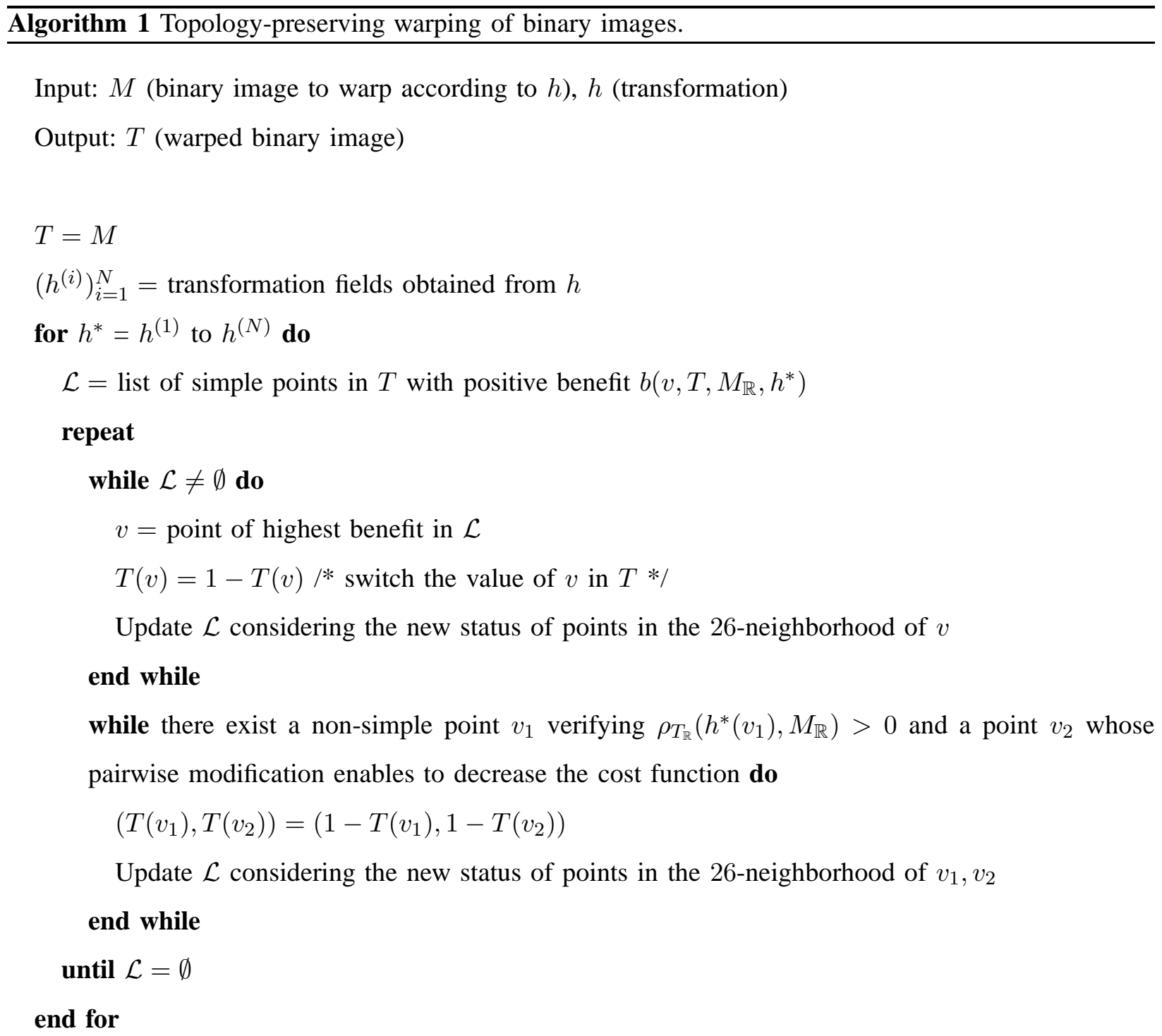




\section{VALIDATION}

\section{A. Atlas-based segmentation: application to skull segmentation from CT data}

One of the main applications of the proposed approach - or similar ones - is atlas-based segmentation [23]. Such methods rely on a binary model $M: E_{\mathbb{Z}}^{M} \rightarrow\{0,1\}$ of given structures of interest which has been obtained from the preliminary segmentation of an "anatomical reference" image $R: E_{\mathbb{Z}}^{M} \rightarrow \mathbb{R}$. When attempting to detect the same structures of interest in a new image $I: E_{\mathbb{Z}}^{T} \rightarrow \mathbb{R}$, the first step consists in estimating a transformation $h: E_{\mathbb{R}}^{T} \rightarrow E_{\mathbb{R}}^{M}$ by registering $R$ onto $I$. The structures of interest in $I$, denoted $T$, are then obtained by transforming $M$ according to $h$.

Following this methodological framework, skull segmentation then consists in deforming a pre-processed skull template $M$ associated to a reference CT image $R$. The skull template considered in this work has been created from a CT image $R$ according to the following paradigm. First, a segmentation has been carried out by performing a simple thresholding on $R$. This first rough segmentation has been modified by an expert: he removed the parts which did not present any interest for the desired applications (essentially structures located on its inferior side), he improved the quality of the segmentation and checked finally that the image was composed of one OCC. Then, a hole-closing algorithm [10] was applied in order (i) to remove (i.e. to fill) the cavities, and $(i i)$ to localize the handles, thus enabling to preserve (resp. to fill) the ones corresponding (resp. not corresponding) to relevant anatomical structures. It has to be noticed that the erroneous handles were generally one voxel-thick and located in thin structures. The template is composed of one connected component, and has no cavity. However, it presents ten handles (its Euler characteristic is equal to -9) corresponding to specific anatomical structures (e.g. foramen magnum, zygomatic arches, optic canals, external auditory meatus, etc., see Fig. 5) leading to a complex topology. From a geometrical point of view, this template is composed of "surfacic structures" of varying thicknesses (from several millimeters/voxels) to one millimeter (then leading to one voxel-thin surfaces, and justifying the use of the 26-adjacency for its topological modeling), for instance near the eyes and the nose.

The segmentation of a new image $I$ is obtained by warping the template $M$ according to a 3-D transformation $h$ estimated by registering $R$ onto $I$. Since the discrete topological properties of the segmentation are expected to be the same than those of $M$, the use of a topology-preserving warping is here of great interest.

It has to be noticed that the proposed segmentation strategy requires $(i)$ to compute a correct deformation field $h$, and $(i i)$ to correctly deform $M$ according to $h$. In the proposed experiments, the deformation 


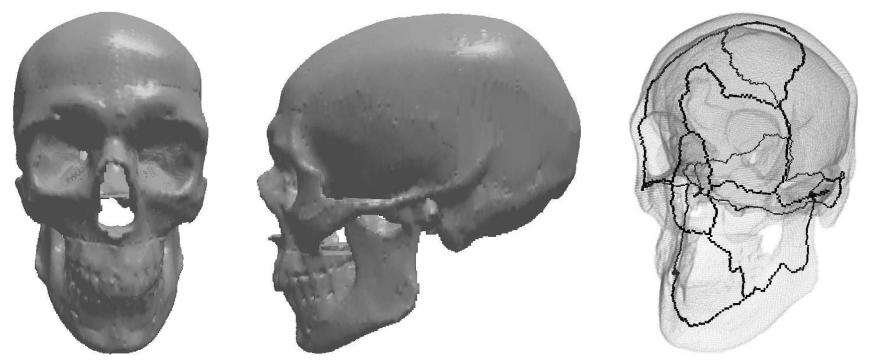

Fig. 5. Skull template used in the proposed application. Left, middle: front and profile view of the template. Right: template visualized with its topological skeleton.

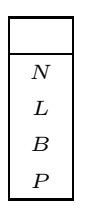

\begin{tabular}{|c|c|c|c|c|}
\hline$E_{0}$ & $E_{1}$ & $E_{2}$ & $E_{3}$ & $d_{\max }$ \\
\hline 100 & 0 & 0 & 0 & 0 \\
99.8742 & 0.0865 & 0.0393 & 0 & 0.99 \\
99.5248 & 0.2357 & 0.2386 & 0.0009 & 30.98 \\
99.9944 & 0.0043 & 0.0013 & $10^{-5}$ & 1.86 \\
\hline
\end{tabular}

\begin{tabular}{|c|c|c|}
\hline$b_{0}$ & $b_{1}$ & $b_{2}$ \\
\hline $1.33 \pm 0.59$ & $109 \pm 51.0$ & $150 \pm 71.0$ \\
$1.06 \pm 0.25$ & $24.8 \pm 8.00$ & $7.20 \pm 2.80$ \\
$1.00 \pm 0.00$ & $10.0 \pm 0.00$ & $0.00 \pm 0.00$ \\
$1.00 \pm 0.00$ & $10.0 \pm 0.00$ & $0.00 \pm 0.00$ \\
\hline
\end{tabular}

\section{TABLE I}

COMPARISON ACCORDING TO GEOMETRICAL $\left(E_{i}\right.$, AND $\left.d_{\max }\right)$ AND TOPOLOGICAL $\left(b_{i}\right)$ CRITERIA OF THE PROPOSED METHOD $(P)$ WITH THE NEAREST NEIGHBOR INTERPOLATION $(N)$, THE LINEAR INTERPOLATION WITH THRESHOLDING $(L)$, AND THE METHOD PROPOSED IN [20] $(B)$. $E_{0}$ : RATIO $(\%)$ OF POINTS $v$ FOR WHICH $\rho_{T_{\mathbb{R}}}\left(h(v), M_{\mathbb{R}}\right)=0, E_{1}$ FOR WHICH $0<\rho_{T_{\mathbb{R}}}\left(h(v), M_{\mathbb{R}}\right) \leq 0.5, E_{2}$ FOR WHICH $0.5<\rho_{T_{\mathbb{R}}}\left(h(v), M_{\mathbb{R}}\right) \leq 1$, AND $E_{3}$ FOR WHICH $\rho_{T_{\mathbb{R}}}\left(h(v), M_{\mathbb{R}}\right)>1\left(\rho_{T_{\mathbb{R}}}\right.$ IS GIVEN IN VOXEL); $d_{\text {max }}$ : MAXIMAL VALUE OF $\rho_{T_{\mathbb{R}}}\left(h(v), M_{\mathbb{R}}\right)$ FOR THE 15 CASES; $b_{0}$ (RESP. $b_{1}, b_{2}$ ): NUMBER OF OBJECT CONNECTED COMPONENTS (RESP. HANDLES, CAVITIES).

field $h$ has been estimated using the continuous topology-preserving registration strategy described in [24] ( $h$ is a complex transformation modeled using first order B-spline functions with approximately 750000 parameters).

Four methods have been considered to deform $M$ according to $h$ : the proposed method $(P)$, the nearest neighbor interpolation $(N)$, the linear interpolation followed by a thresholding at value $0.5(L)$, and the method proposed in [20] $(B)$. All these methods have been applied on a database composed of 15 isotropic $256^{3} \mathrm{CT}$ images of millimetric resolution. Segmentation results are strongly related to the accuracy of the estimation of $h$. Consequently, the methods are not compared here in terms of segmentation accuracy, but in terms of topological and geometrical adequacy of their result w.r.t. $h$. 


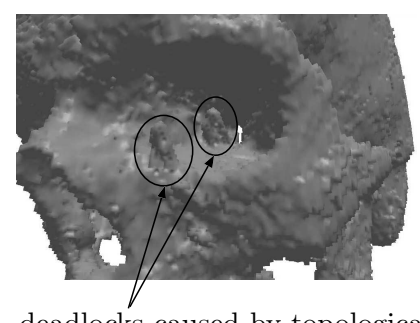

deadlocks caused by topological constraints

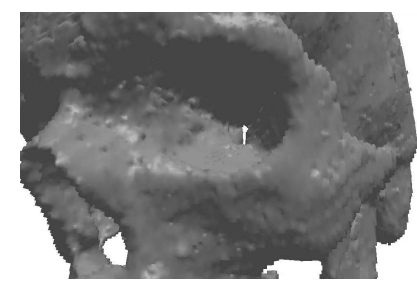

Fig. 6. Segmentation results obtained with the method of [20] $(B$, left), the proposed method $(P$, middle) and the nearest neighbor interpolation ( $N$, right) (see text). Note that the surfaces are visually noisy since the real discrete results are visualized here without any post-processing operation such as mesh generation.

\section{B. Results}

In order to assess the quality of the methods from a topological point of view, the Betti numbers $b_{i}$ $(i \in[0,2])$ are computed for the 15 results $T$ obtained for each method (see Table. I).

The Betti numbers are defined as the ranks of the different homology groups of an object [25]. In 3-D, $b_{0}, b_{1}$, and $b_{2}$ correspond to the number of OCCs, the number of handles, and the number of cavities (or equivalently the number of BCCs-1), respectively. It can be observed that $P$ and $B$ are the only methods guaranteeing topology preservation: the topology is strongly altered with $N$ and $L$, leading to connected component splittings, handle and cavity generations, etc. For instance, the method $N$ generates an average number of 150 undesired cavities in the segmentation result.

From a geometrical point of view, a quantitative comparison is provided by computing $\rho_{T_{\mathbb{R}}}$ for each method ( $\rho_{T_{\mathbb{R}}}$ is given in voxel). This computation requires a one-to-one mapping $\phi$ between the CCs of $M$ and those of $T$, which is not ensured for the methods $N$ and $L$. Since $M$ is composed here of exactly one OCC and one BCC, this limitation can be easily overcome: voxels $v$ for which $T(v)=1$ are associated with the OCC, and the other ones to the BCC. Table I provides for each method the ratios of points for which $\rho_{T_{\mathbb{R}}}=0\left(E_{0}\right), 0<\rho_{T_{\mathbb{R}}} \leq 0.5\left(E_{1}\right), 0.5<\rho_{T_{\mathbb{R}}} \leq 1\left(E_{2}\right)$, and $\rho_{T_{\mathbb{R}}}>1\left(E_{3}\right)$, and the maximal distance $d_{\max }$ defined as the maximal value of $\rho_{T_{\mathbb{R}}}$ for the 15 cases.

As expected, $\rho_{T_{\mathbb{R}}}\left(h(v), M_{\mathbb{R}}\right)=0$ for all voxels $v$ when considering the $N$ method (see Section II-B). The results obtained with the $P$ and the $L$ methods show that more points are well located using the $P$ method $\left(E_{0}=99.9944 \%\right.$ with $P$, and only $99.8742 \%$ with $\left.L\right)$. However, since topology preservation induces here geometric constraints, the ratio of points for which $\rho_{T_{\mathbb{R}}}>1$ is equal to $10^{-5} \%$ with $P$ (and 0 with $L$ ). This ratio is still very low for $P$ and corresponds to 26 voxels on the 15 considered cases. 
Note finally that the maximal distance $d_{\max }$ is a little bit higher (but still low) for the proposed method $\left(d_{\max }=1.86\right.$ for $P$ and 0.99 for $\left.L\right)$. More precisely, with respect to the method $P$, the maximal values of $\rho_{T_{\mathbb{R}}}\left(h(v), M_{\mathbb{R}}\right)$ for the 15 images are, from the lowest to the highest: $0.80,0.84,0.85,0.93,0.93,0.97$, $0.97,0.98,1.02,1.62,1.62,1.74,1.79,1.84,1.86$. Thus, we can conclude that the geometrical adequacy of the results is quite similar for $P, N$ and $L$, but among these three methods, only $P$ can correctly handle topology.

As mentioned in Section I, $B$ enables to preserve the topology but may fail to converge to the geometrically desired solution. This is illustrated by the fact that $d_{\max }$ is equal to 30.98 for $B$ (moreover, the maximal values of $\rho_{T_{\mathbb{R}}}\left(h(v), M_{\mathbb{R}}\right)$ for the 15 images are, from the lowest to the highest: 1.9, 3.4, $3.7,4.4,4.9,6.5,6.6,10.7,12.4,15.5,15.6,17.4,21.9,26$ and 30.98). Furthermore, the values $E_{i}$ show that $B$ is hardly accurate. One could argue that such a claim is biased since quantification of the results is based on the proposed cost function. However, as very good results are obtained with the $N$ and $L$ approaches, this cost function can reasonably be considered as meaningful.

The above analysis emphasizes that $P$ outperforms the existing -topology-preserving- method while still leading to satisfactory geometrical adequacy of the results, similar to those obtained for non topologypreserving methods. The conclusions of this study are illustrated by Fig. 6, which presents a typical result for $B, P$ and $N$. An erroneous handle appears with $N$ (remember that "standard" methods do not preserve the topology) whereas $B$ does not converge to the geometrically desired solution, which results in the apparition of two artifacts. Such artifacts, which are very similar to lumps[26] (lumps are topological artifacts, generally presenting a complex shape, which can appear during topology-preserving monotonic transformation of binary objects of $\mathbb{Z}^{n}$ for $n \geq 3$ ) are consequences of topological deadlocks that occurred during the estimation of the digital homeomorphism $h_{d}$ ( $h$ is approximated by a digital homeomorphism $h_{d}$, see Section I), thus preventing to converge to the desired solution.

\section{Influence of the different steps of the proposed approach}

As it has been mentioned in Subsection II-D, the introduction of pairwise modifications, and the decomposition of the transformation into small displacements are two steps which are required to achieve good convergence properties. To investigate how critical are these two points, three new experiments have been carried out. We denote by $P W M$ the pairwise modification step and by $S D$ the use of small displacement, namely, the fact to consider sequentially the intermediate deformation fields $h^{(1)}, h^{(2)}, \ldots$, $h^{(N)}$ estimated from $h$. Three different methods, $P_{1}, P_{2}$, and $P_{3}$, are derived from the proposed method $P$ as follows: the $P W M$ and the $S D$ steps are not used in $P_{1}$. Only the $P W M$ step is used in $P_{2}$ and only 
the $S D$ step is used in $P_{3}$. These three methods have been applied on the aforementioned database. The geometrical adequacy of the results obtained with $P, P_{1}, P_{2}$, and $P_{3}$ are provided in Table II. Criteria related to topology are not presented here since all these methods preserve the topology.

\begin{tabular}{|c|c|c|c|c|c|}
\hline & $E_{0}$ & $E_{1}$ & $E_{2}$ & $E_{3}$ & $d_{\max }$ \\
\hline$P$ \\
\hline$P_{1}$ \\
$P_{2}$ \\
$P_{3}$ \\
\hline 99.9944 & 0.0043 & 0.0013 & $10^{-5}$ & 1.86 \\
99.9326 & 0.0122 & 0.0216 & 0.0336 & 15.06 \\
99.9522 & 0.0098 & 0.0157 & 0.0223 & 13.01 \\
99.9757 & 0.0027 & 0.0035 & 0.0181 & 18.57 \\
\hline
\end{tabular}

TABLE II

Comparison ACCORding to Geometrical $\left(E_{i}\right.$, AND $\left.d_{\max }\right)$ CRiteria of the Proposed method $(P)$, With $P_{1}, P_{2}$, AND $P_{3}$ (SEE TEXT). THE CRITERIA ARE THE SAME THAN THOSE USED IN TABLE I.

We can first notice that results are much less satisfactory without the $P W M$ and the $S D$ steps $\left(P_{1}\right)$ when compared with the original method $(P)$. Indeed, the ratio of points for which $\rho_{T_{\mathbb{R}}}>1$, and $d_{\max }$ have largely increased $\left(E_{3}=0.0336 \%\right.$ for $P_{1}$ v.s. $10^{-5} \%$ for $P$, and $d_{\max }=15.06$ for $P_{1}$ v.s. 1.86 for $P$ ). Moreover, with the $P_{1}$ approach, topological deadlocks can be observed in all images since the maximal values of $\rho_{T_{\mathbb{R}}}\left(h(v), M_{\mathbb{R}}\right)$ for the 15 images range from 8.87 to 15.06 . These results show clearly the great importance of the $P W M$ and of the $S D$ steps.

One can then notice that the results obtained using $P_{2}$ and $P_{3}$ are improved compared to those obtained using $P_{1}$. However, even if the ratio of points for which $\rho_{T_{\mathbb{R}}}>1$ has decreased using $P_{2}$ and $P_{3}$ with respect to $P_{1}$, it remains largely greater than the one obtained with $P$. Moreover, topological deadlocks are observed with $P_{2}$ and $P_{3}$ for each considered image: the maximal values of $\rho_{T_{\mathbb{R}}}\left(h(v), M_{\mathbb{R}}\right)$ for the 15 images range from 5.09 to 15.06 for $P_{2}$ and from 7.98 to 18.57 for $P_{3}$. These results emphasize the fact that the $P W M$ and the $S D$ steps are both required to obtain good results. This is not a surprising conclusion since the $P W M$ step aims at escaping from local minima or from deadlock situations which may appear during the dynamical scheme while the $S D$ step aims at avoiding deadlock situations by guiding the discrete dynamical process.

The influence of the parameter $\epsilon$ used for decomposing the transformation is also investigated. Four different values are used: $\epsilon=0.25\left(P_{\epsilon=0.25}\right), \epsilon=0.5\left(P=P_{\epsilon=0.5}\right), \epsilon=1\left(P_{\epsilon=1}\right)$, and $\epsilon=5\left(P_{\epsilon=5}\right)$. Results are given in Table III.

Results obtained for $P_{\epsilon=0.25}$ and for $P_{\epsilon=0.5}$ are very similar, while those obtained for $\epsilon=1$ are 


\begin{tabular}{|c|c|c|c|c|c|}
\hline & $E_{0}$ & $E_{1}$ & $E_{2}$ & $E_{3}$ & $d_{\max }$ \\
\hline$P_{\epsilon=0.25}$ & 99.9980 & 0.0015 & 0.0005 & $10^{-5}$ & 1.86 \\
\hline$P_{\epsilon=0.5}$ & 99.9944 & 0.0043 & 0.0013 & $10^{-5}$ & 1.86 \\
\hline$P_{\epsilon=1}$ & 99.9977 & 0.0016 & 0.0006 & 0.0001 & 4.76 \\
\hline$P_{\epsilon=5}$ & 99.9897 & 0.0028 & 0.0023 & 0.0052 & 18.59 \\
\hline
\end{tabular}

TABLE III

COMPARISON ACCORDING TO GEOMETRICAL $\left(E_{i}\right.$, AND $\left.d_{\max }\right)$ CRITERIA OF THE PROPOSED METHOD WITH DIFFERENT Values of $\epsilon: P_{\epsilon=0.25}, P=P_{\epsilon=0.5}, P_{\epsilon=1}, P_{\epsilon=5}$ (SeE teXt). The Criteria are the Same than those used in Table I.

slightly deteriorated: $E_{3}=0.0001 \%$ for $P_{\epsilon=1}$ v.s. $10^{-5} \%$ for $P_{\epsilon=0.5}$, and $d_{\max }=4.76$ for $P_{\epsilon=1}$ v.s. 1.86 for $P_{\epsilon=0.5}$. More precisely, results are largely deteriorated for only one image whereas results are relatively satisfactory for the others. Indeed, the maximal values of $\rho_{T_{\mathbb{R}}}\left(h(v), M_{\mathbb{R}}\right)$ for the 15 images are, from the lowest to the highest: $0.80,0.93,1.03,1.17,1.36,1.47,1.53,1.60,1.62,1.69,1.79,1.82$, 2.27, 2.58 and 4.76. On the contrary, when $\epsilon$ is set to 5 , some topological deadlocks appear in most of the images: the maximal values of $\rho_{T_{\mathbb{R}}}\left(h(v), M_{\mathbb{R}}\right)$ for the 15 images range from 2.01 to 18.59 , and the maximal value of $\rho_{T_{\mathbb{R}}}\left(h(v), M_{\mathbb{R}}\right)$ is greater than 10 for six cases.

At the sight of these results, the setting $\epsilon=0.5$ appears to be a relevant choice.

\section{Discussion}

The previous section highlights the good performance of the proposed method. However, as for any method based on a dynamic scheme, which attempts to minimize an energy function, there is always a risk to converge to a local minimum. Indeed, it is possible to design ad hoc examples leading the method to converge to such a local minimum.

A first example, illustrated in Fig. 7, highlights one deadlock situation of the proposed approach. In this example, the image $M$ to warp is composed of two OCCs of one voxel, namely, $v_{1}$ and $v_{2}$. Let us suppose that the considered topology-preserving deformation field $h$ verifies $h\left(v_{1}\right)=v_{2}$, and $h\left(v_{2}\right)=v_{1}$ (for example, $h$ can be a rotation of $\pi$ radians whose center $S$ is the middle point between $v_{1}$ and $v_{2}$ ). Three intermediate transformations $h^{(i)}(i \in[1,3])$ are then computed from $h$. When considering $h^{(1)}$, the proposed approach converges from $M$ to $T_{\text {res }}$. When considering $h^{(2)}$ (and, a fortiori, $h^{(3)}$ and $h$ ), there is no simple point with positive benefit value. Consequently, $T_{\text {res }}$ corresponds to a local minimum which will be the result of the method for this example. The result $T_{r e s}$, although topologically correct 


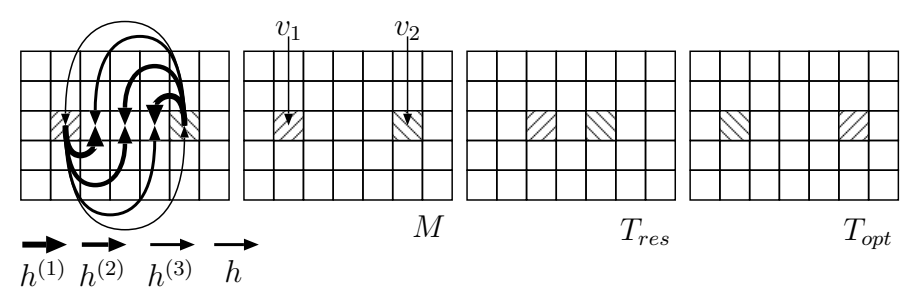

Fig. 7. Example illustrating a deadlock situation of the proposed approach. From left to right: representation of $h$ and of the intermediate transformations $h^{(i)}(i \in[1,3]) ; M$ (image to warp), $T_{\text {res }}$ (obtained result), and $T_{o p t}$ (desired result). The proposed approach converges here to a local minimum since the discrete dynamical scheme cannot follow the path provided by the intermediate transformations, due to topological constraints.

is however geometrically very far from the expected result $T_{\text {opt }}$. The main reason explaining the failure of the process is that $h^{(2)}$ is a many-to-one mapping: $h^{(2)}\left(v_{1}\right)=h^{(2)}\left(v_{2}\right)$. Since the dynamical process is constrained to preserve topology, voxels cannot be moved according to $h^{(2)}$. This is a consequence of the construction of the intermediate transformations $h^{(i)}$ proposed in Section II, that may possibly not preserve topology. We can notice that this limitation also holds in the approach of [20] where the movement of simple point $v_{i}$ is guided by choosing the closest 6 -adjacent neighbor voxel $v_{j}$ along the direction of the vector $h\left(v_{i}\right)-h_{d}\left(v_{i}\right)$ ( $h$ is approximated by a digital homeomorphism $h_{d}$, see Section I).

In order to illustrate such a phenomenon on a "real" case, let us consider the skull reference image $M$ used in Section III, and for $h$, a rotation of $\pi$ radians around an axis parallel to the $x$-axis and passing through the middle of the image. Broadly speaking, all points may collapse onto the discrete line corresponding to this rotation axis during the dynamical scheme. Results obtained with [20] and the proposed method are illustrated in Fig. 8. As expected, both methods do not converge to the desired solution. The way in which the method $B$ fails can be at first sight surprising. The artifacting lines that are oriented in the direction of the axis of rotation are in fact a side effect of considering a discrete deformation field to warp the binary image toward the desired solution. For instance, when considering a voxel $v$ located at a corner of the image, the corresponding continuous deformation vector $h(v)$ associates this voxel with the opposite corner $v^{\prime}$ of the image with respect to the axis of rotation. Thus, during the optimization process and provided there is no topological deadlock, the discrete deformation field $h_{d}(v)$ to be estimated will successively associate the voxel $v$ with every voxels located on the line $\left(v v^{\prime}\right)$ until converging to $v^{\prime}$. Consequently, the voxel $v$ will be associated sometimes with background 


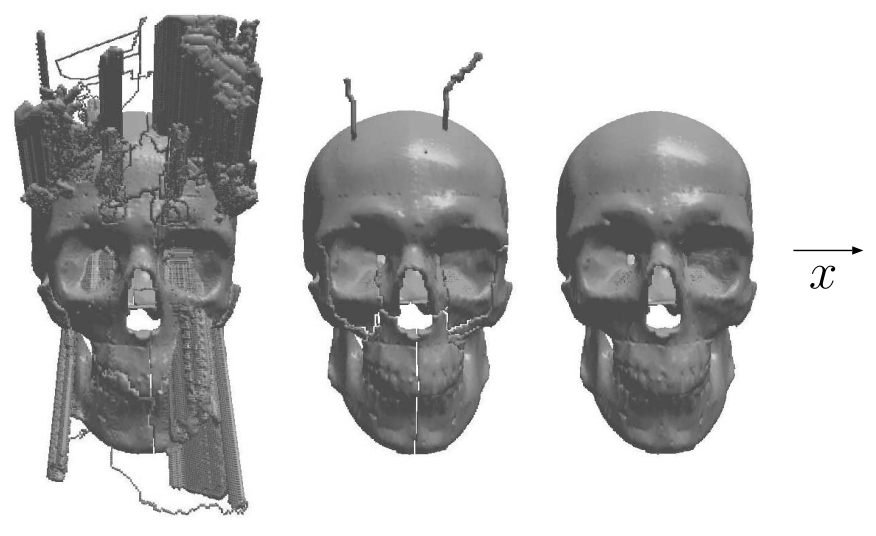

Fig. 8. Results obtained for $h$ corresponding to a rotation of $\pi$ radians with [20] (left), and with the proposed method, by considering a linear decomposition of $h$ (middle) and an ad hoc rotation-based decomposition (right). Results are visualized up to the rotation of $\pi$ radians, in order to look similar to the left part of Fig. 5.

components and sometimes with object components, thus explaining the emergence of theses kinds of "spike". Some of these "spikes" disappear when the discrete deformation succeeds to pass through all the object components, but some of them remain because of topological deadlocks. Concerning the two "spikes" that appear with our method, they result from voxels belonging to the chin that get stuck there because of topological deadlocks.

However, the proposed approach could easily be extended to circumvent this limitation by constraining each transformation $h^{(i)}$ to be a one-to-one mapping. For example, in this example, the $N+1$ intermediate transformations computed from $h$ could be defined as follows: $h_{i}$ is the rotation of $i . \pi / N$ radians around the rotation axis with $N$ being the smallest integer which enables to verify Eq. 7. The result obtained with a rotation-based decomposition is presented in the right part of Fig. 8 and exactly corresponds to the expected solution.

In a more general fashion, an efficient way to deal with transformations which are not correctly modeled by a linear decomposition is to consider the transformation as the composition of several "elementary" ones: $h=h_{1} \circ h_{2} \circ \ldots h_{k}$, where each $h_{t}$ belongs to a specific class of transformation such as translations, rotations, scalings, etc. Adapted strategies could then be used to estimate the intermediate transformations $\left(h_{t}^{i}\right)_{i=1}^{N_{t}}$ for each $h_{t}$, such as the one proposed above for axial rotation along a principal axis which enables, by composition, to model any rotation, or such as the framework presented in [27] for estimating large deformation diffeomorphisms, which aims at defining a geodesic pathway in the manifold of diffeomorphisms. It has also to be noticed that the transformation of a whole discrete image 
by $(i)$ a translation by a vector $(x, y, z) \in \mathbb{Z}^{3},(i i)$ a rotation of $k . \pi / 2(k \in \mathbb{Z})$ along one of the 3 principal axes of $\mathbb{Z}^{3}$, and (iii) a scaling by a factor $k \in \mathbb{N}^{*}$ (i.e. an oversampling), trivially preserve the topology of the image. Following this remark, a large rotation (e.g., of $5 \pi / 6$ radians) could be divided into two rotations: a -possibly large- first one (of $\pi$ radians) preserving the topology, and a small second one (of $-\pi / 6$ radians).

Unfortunately, there is however no guarantee that the discrete dynamical process may "follow the path" generated by the successive one-to-one mappings. In the example depicted in Fig. 9, the image $M$ is composed of two OCCs and is warped according to a rotation of $\pi$ radians around the point $O$. The sequence of one-to-one mappings $\left(h^{(i)}\right)_{i=1}^{N}$ which verify the properties of Eq. 7 can easily be

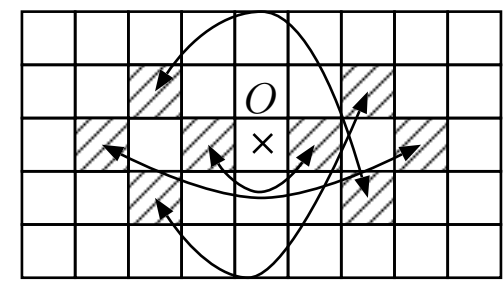

Fig. 9. Image to warp according to a rotation of $\pi$ radians around $O$. Even if it is possible to find a sequence of one-to-one mappings $\left(h^{(i)}\right)_{i=1}^{N}$, the dynamical process cannot converge to the desired solution (see text).

computed thanks to the aforementioned strategy. However, the dynamical process, which is discrete by definition, will not be able to follow the associated path. Indeed, the desired result, which consists, in "exchanging" the two OCCs and their respective handles, cannot be obtained by adding and/or removing simple points from $M:(i)$ the topological complexity of the two OCCs does not enable to "reduce" them without altering their topology and (ii) the image is not large enough to enable the two OCCs with their associated handles to move simultaneously towards their respective targets. A solution could be to enlarge the image by adding voxels on its border. However, despite such considerations, in the general case, the main difficulty remains the computation of a sequence $\left(h^{(i)}\right)_{i=1}^{N}$ of one-to-one mappings such that the discrete scheme is able to follow the corresponding paths. In practice, this estimation will clearly depend on the topological complexity of the object to warp. For instance, the local Jacobian should be large in areas where topological properties are complex, and small in areas with "simple" topological properties. This is clearly a very difficult task, and probably an open problem.

The considerations exposed in this section, although not enabling to solve all the problems induced by the difficulty to decompose any deformation fields into a sequence of one-to-one mappings, enable 
to efficiently deal with a large class of deformations, and to significantly reduce the risk of erroneous convergence of the proposed method to local minima. Nonetheless, the linear decomposition of the deformation field has revealed to provide satisfactory results in the context of our application.

Finally, the experiments described throughout the paper were performed on a $2.4 \mathrm{GHz}$ dual-core Pentium workstation. The overall procedure was implemented in $\mathrm{C} / \mathrm{C}++$. On average, approximately half an hour is required to transform an image of size $256 \times 256 \times 256$ according to a deformation field. This may appear computationally expensive compared to standard interpolation methods such as the nearest neighbor interpolation which requires a few seconds. However, this time is acceptable compared to the approximatively 3 hours required in our application to estimate a deformation field from two CT-images.

\section{CONCLUSiON}

A new method for warping a binary image in a discrete topology-preserving fashion according to a continuous topology-preserving transformation has been proposed. This method is among the first ones devoted to fuse continuous topology, i.e. the topology related to the actual semantic of the continuous warped image, with discrete topology, i.e. the topology which can effectively be handled in a digital image. Experiments have been conducted in the context of atlas-based skull segmentation. Standard interpolation methods such as nearest-neighbor and linear interpolation have been shown to alter the discrete topological properties of the warped images (appearance of handles and cavities). In addition, the proposed method has been compared with an alternative method of the literature [20] that also ensures topology preservation and has been shown to geometrically better converge to the desired solution: the maximal geometrical error observed with the proposed approach has never exceeded $2 \mathrm{~mm}$ while the alternative method has led to error higher that $30 \mathrm{~mm}$ for some experiments. These results emphasize the interest of the proposed approach which may be of interest for other applications in 3-D medical image analysis.

Further works will consist in extending this method to label images. It can be noticed that some methods have already been proposed to modify label images while preserving their putative topology [28], [29], [16], [20]. However: $(i)$ in [29], some simplifying hypotheses are used to enable the handling of such label models as multicomponent binary ones; (ii) in [16], the considered theoretical framework is not sufficiently robust to guarantee complete results (the result of the segmentation can possibly lead to a partial partition of the image, i.e. some points may possibly be unclassified); and (iii) in [28], [20], the proposed theoretical framework is not compatible with a 2-label (i.e. a binary) image, in particular, it raises several paradoxes related to the Jordan-Brouwer theorem [30]. Note that the correctness of the 
"deformation" part of the method proposed in [20] in the case of -correctly handled- binary images is however not affected by the proposed label image modeling. The extension of the method proposed in this article to the case of label images will first require to develop a sound theoretical framework for modeling and topology-controlled modification of label images, which is, to our knowledge, still an open problem.

\section{REFERENCES}

[1] M. Holden, "A review of geometric transformations for nonrigid body registration," IEEE Transactions on Medical Imaging, vol. 27, no. 1, pp. 111-128, 2008.

[2] T. Lehmann, C. Gonner, and K. Spitzer, "Survey: interpolation methods in medical image processing," IEEE Transactions on Medical Imaging, vol. 18, no. 11, pp. 1049-1075, 1999.

[3] D. Shattuck and R. Leahy, "Automated graph-based analysis and correction of cortical volume topology," IEEE Transactions on Medical Imaging, vol. 20, no. 11, pp. 1167-1177, 2001.

[4] N. Passat, C. Ronse, J. Baruthio, J.-P. Armspach, M. Bosc, and J. Foucher, "Using multimodal MR data for segmentation and topology recovery of the cerebral superficial venous tree," in ISVC'05, 2005, pp. 60-67.

[5] N. Kriegeskorte and R. Goebel, "An efficient algorithm for topologically correct segmentation of the cortical sheet in anatomical MR volumes," NeuroImage, vol. 14, no. 2, pp. 329-346, 2001.

[6] X. Han, C. Xu, U. Braga-Neto, and J. Prince, "Topology correction in brain cortex segmentation using a multiscale, graph-based algorithm," IEEE Transactions on Medical Imaging, vol. 21, no. 2, pp. 109-121, 2002.

[7] S. Bischoff and L. Kobbelt, "Isosurface reconstruction with topology control," in PG'02, 2002, pp. 246-255.

[8] F. Ségonne, E. Grimson, and B. Fischl, “Topological correction of subcortical segmentation,” in MICCAI'03, 2003, pp. 695-702.

[9] P.-L. Bazin and D. Pham, “Topology correction using fast marching methods and its application to brain segmentation," in MICCAI'05, 2005, pp. 484-491.

[10] Z. Aktouf, G. Bertrand, and L. Perroton, "A three-dimensional holes closing algorithm," Pattern Recognition Letters, vol. 23, no. 5, pp. 523-531, 2002.

[11] A. Szymczak and J. Vanderhyde, "Extraction of topologically simple isosurfaces from volume datasets," in VIS 2003, 2003, pp. $67-74$.

[12] J.-F. Mangin, V. Frouin, I. Bloch, J. Régis, and J. López-Krahe, "From 3D magnetic resonance images to structural representations of the cortex topography using topology preserving deformations," Journal of Mathematical Imaging and Vision, vol. 5, no. 4, pp. 297-318, 1995.

[13] P. Dokládal, C. Lohou, L. Perroton, and G. Bertrand, "Liver blood vessels extraction by a 3-D topological approach," in MICCAI'99, 1999, pp. 98-105.

[14] X. Han, C. Xu, and J. Prince, "A topology preserving level set method for geometric deformable models," IEEE Transactions on Pattern Analysis and Machine Intelligence, vol. 25, no. 6, pp. 755-768, 2003.

[15] N. Passat, C. Ronse, J. Baruthio, J.-P. Armspach, C. Maillot, and C. Jahn, "Region-growing segmentation of brain vessels: An atlas-based automatic approach,” Journal of Magnetic Resonance Imaging, vol. 21, no. 6, pp. 715-725, 2005.

[16] P.-L. Bazin and D. Pham, "Topology-preserving tissue classification of magnetic resonance brain images," IEEE Transactions on Medical Imaging, vol. 26, no. 4, pp. 487-496, 2007. 
[17] T. Kong and A. Rosenfeld, "Digital topology: Introduction and survey," Computer Vision, Graphics, \& Image Processing, vol. 48, no. 3, pp. 357-393, 1989.

[18] G. Bertrand and G. Malandain, "A new characterization of three-dimensional simple points," Pattern Recognition Letters, vol. 15, no. 2, pp. 169-175, 1994.

[19] Y. Cointepas, I. Bloch, and L. Garnero, "Joined segmentation of cortical surface and brain volume in MRI using a homotopic deformable cellular model,' in 3DIM'99, 1999, pp. 240-248.

[20] P.-L. Bazin, L. Ellingsen, and D. Pham, "Digital homeomorphisms in deformable registration," in IPMI'07, 2007, pp. 211-222.

[21] S. Faisan, N. Passat, V. Noblet, R. Chabrier, and C. Meyer, "Topology preserving warping of binary images. Application to atlas-based skull segmentation," in MICCAI'08, 2008, pp. 211-218.

[22] T. Y. Kong, “Topology-preserving deletion of 1's from 2-, 3- and 4-dimensional binary images,” in DGCI'97, 1997, pp. $3-18$.

[23] B. Dawant, S. Hartmann, J.-P. Thirion, F. Maes, D. Vandermeulen, and P. Demaerel, “Automatic 3-D segmentation of internal structures of the head in MR images using a combination of similarity and free-form deformations: Part I, methodology and validation on normal subjects," IEEE Transactions on Medical Imaging, vol. 18, no. 10, pp. 902-916, 1999.

[24] V. Noblet, C. Heinrich, F. Heitz, and J.-P. Armspach, “3-D deformable image registration: a topology preservation scheme based on hierarchical deformation models and interval analysis optimization," IEEE Transactions on Image Processing, vol. 14 , no. 5, pp. 553-556, 2005.

[25] P. Giblin, Graphs, surfaces and homology. Chapman and Hall, 1981.

[26] N. Passat, M. Couprie, and G. Bertrand, "Minimal simple pairs in the 3-D cubic grid," Journal of Mathematical Imaging and Vision, vol. 32, no. 3, pp. 239-249, 2008.

[27] S. Joshi and M. Miller, "Landmark matching via large deformation diffeomorphisms," IEEE Transactions on Image Processing, vol. 9, no. 8, pp. 1357-1370, 2000.

[28] F. Poupon, J.-F. Mangin, D. Hasboun, C. Poupon, I. Magnin, and V. Frouin, "Multi-object deformable templates dedicated to the segmentation of brain deep structures," in MICCAI'98, 1998, pp. 1134-1143.

[29] S. Miri, N. Passat, and J.-P. Armspach, "Topology-preserving discrete deformable model: Application to multi-segmentation of brain MRI,' in ICISP'08, 2008, pp. 67-75.

[30] E. Spanier, Algebraic Topology. New York: McGraw-Hill, 1966. 